M Kamiński ${ }^{1}$, J Kippen ${ }^{1}$, A Gomulska ${ }^{1}$, J Smyrak ${ }^{1}$, M Karolewski² $^{2}$ L Bielawska ${ }^{3}$, E Wysocka ${ }^{3}$, M Cymerys $^{1}$

${ }^{1}$ Department of Internal Medicine, Endocrinology, Diabetology, Poznan University of Medical Sciences, Grunwaldzka 16/18 , 60-780 Poznań, Poland

${ }^{2}$ Cardiology Ward, John Paul II District Hospital in Trzcianka, 64-980 Trzcianka, Poland

${ }^{3}$ Department of Laboratory Diagnostics, Poznan University of Medical Sciences, Szamarzewskiego str. 82/84, 60-569 Poznan, Poland

\title{
Myonectin serum concentration changes after short-term physical activity among young, healthy people
}

\author{
Corresponding author: \\ Mikołaj Kamiński \\ Department of Internal Medicine, \\ Endocrinology, Diabetology, Poznan \\ University of Medical Sciences, \\ Grunwaldzka 16/18 \\ 60-780 Poznań, Poland \\ phone no/fax: + 48618547828 \\ e-mail: mikolaj.w.kaminski@gmail.com
}

Medical Research Journal 2019; Volume 4, Number 1, 41-45 DOI: 10.5603/MRJ.a2019.0002 Copyright (C) 2019 Via Medica ISSN 2451-2591

\begin{abstract}
Background: Myonectin is a myokine secreted by skeletal muscles in response to physical activity (PhA) in rodents. It was shown that myonectin may be positively associated with insulin resistance parameters. The aim of the study was to evaluate changes in the concentration of myonectin after short-term PhA. Methods: A total of 29 young, healthy volunteers, were included in the study. Each participant completed a life-style questionnaire, underwent a physical examination with anthropometric measurement followed by a treadmill test according to theBruce protocol. Blood samples were collected before and after PhA. An ELISA Assay was used to investigate the myonectin serum level.

Results: The myonectin serum level did not change significantly after $\mathrm{PhA}$ (0.62[0.14-2.9] vs. 1.08[0.15-2.44] $\mathrm{ng} / \mathrm{ml} ; \mathrm{p}=0.84)$. Before PhA the myonectin serum level differed significantly between men and women (respectively: $3.92[2.24-5.30]$ vs. 0.56[0.15-1.75] $\mathrm{ng} / \mathrm{ml} ; \mathrm{p}=0.02$ ). Before $\mathrm{PhA}$ it had a positive association with weight, BMI, serum creatinine and uremic acid $(p<0.05)$. The change in the level of myonectin serum after PhA had negative associations with weight, BMI, fasting insulin level and HOMA-IR $(p<0.05)$. Conclusions: Myonectin serum concentration does not change after short-term physical activity among young, healthy people. Changes in the myonectin serum level after short-term physical activity may be associated with fasting insulin resistance.

Keywords: myonectin, physical, activity, insulin, resistance, treadmill, homa-ir
\end{abstract}

Med Res J 2019; 4 (1): 41-45

\section{Introduction}

Myonectin (CTRP15/C1QTNF/erythroferrone) is a novel myokine secreted by skeletal muscles in response to physical activity $(\mathrm{PhA})$ and the rise of glucose and free fatty acids (FFA) in rodents [1-2]. Myonectin increases the expression of proteins transporting FFA through hepatocytes and adipocyte cell membranes, causing increasing serum FFA uptake [3]. Toloza et al. reported that myonectin level is associated with insulin resistance (IR) in non-diabetic adults [4]. Lim et al. observed that long-term, regular PhA decreases both the myonectin serum level and IR measured by the homeostasis model assessment [5].

It is known that short-term $\mathrm{PhA}$ transiently increases serum levels of glucose, FFA and IR ${ }^{6}$. In this context, it may be suspected that myonectin serum level should increase efter short-term PhA in humans. Currently, no study has yet investigated changes in myonectin serum level after short-term PhA in human adults.

The aim of the study was to evaluate changes in the concentration of myonectin after short-term physical activity.

\section{Materials and Methods}

Design

Cross-sectional, single-centre study.

\section{Data Collection}

The study group consisted of volunteers, young healthy people. All patients were informed about the study and signed an informed consent form. The study 
protocol was approved by the local Ethic Committee (Bioethics Committee of Poznan University of Medical Sciences).

\section{Inclusion and exclusion criteria}

All patients who met the following criteria were included: age between 18-26, visitworklab in fasting state, voluntarily signed a consent form. Exclusion criteria were as follows: metabolic syndrome, acute infection, mental disorder, congenital disease, unstable chronic disease, general exclusion criteria for a treadmill test.

\section{Clinical and laboratory data}

The worklab visit took place in the morning hours (8:00-10:00 AM). All participants had been informed not to eat, drink or smoke 8 hours prior to attendance. All individuals completed a questionnaire including details of sex, age, smoking status and The International Physical Activity Questionnaire (IPAQ) - a validated questionnaire used to assess weekly $\mathrm{PhA}$ expressed as a total weekly metabolic equivalent of the task (MET) [7]. The patients underwent a complete physical examination with anthropometric measurements (weight, height, waist circumference) and blood pressure check. Blood pressure was measured three times by the Korotkoff method in the sitting position, after 10 minutes rest, using a mercury manometer. Blood pressure was additionally measured after 6 minutes of treadmill test. Height and weight were measured using the same medical scales for all patients. Weight was measured to an accuracy of $100 \mathrm{~g}$ and height to $0,5 \mathrm{~cm}$. BMI was calculated from the following equations: $\mathrm{BMI}=$ weight / squared height [kg/m2].

Blood samples were collected in a fasting state using the S-Monovette blood collection system. Serum glucose, serum insulin, creatinine, uremic acid and lipid profile were assessed according to standard laboratory protocols. After the first blood sample collection and before the treadmill test, participants ate breakfast. 6 minutes after the treadmill test a second blood sample was obtained to assess myonectin serum level after PhA. To estimate myonectin serum concentration Human (CTRP15/Myonectin) ELISA Kit (EIAab, China) was used. All steps of the assay were done according to the manufacturer's instructions. Changes in the myonectin serum level were defined as the difference between myonectin serum level after and before physical activity. HOMA-IR was calculated on the basis of the equation [8]:

HOMA -IR $=($ Glucose $*$ Insulin $) / 22.5$

Glucose in $\mathrm{mmol} / \mathrm{l}$, Insulin in $\mu \mathrm{lU} / \mathrm{ml}$.

Treadmill tests were performed by a physician in accordance with the Bruce protocol on Cardiotest1612 (Aspel, Zabrzów, Poland) [9]. For inter- pretation of the treadmill test and counting MET the treadmill manufacturer (Aspel, Zabrzów, Poland) software was used.

\section{Statistical analysis}

Statistical analysis was performed with STATISTICA 12.0 (StatSoft, USA). The normality of variable distributions was tested using the Shapiro-Wilk test. For descriptive analysis, Wilcoxon test was used to compare the results before and after the treadmill test; the Mann-Whitney was performed to compare men and women. For comparison of binary variables, Fisher's exact test was used. Differences with a p-value $<0.05$ were considered statistically significant.

\section{Results}

The study group consisted of 29 participants (male $=10 ; 34.5 \%$ ), the features of the group are presented in Table 1. All participants had myonectin serum levels within the assay range $(0.05-15 \mathrm{ng} / \mathrm{ml})$. To compare myonectin serum levels and other features between men and women the Mann-Whitney $U$ test and Fisher's exact test were used.

Differences between myonectin serum level, heart rate, systolic and diastolic blood pressure before and after physical activity were presented in Table 2 . The $\mathrm{R}$ Spearman rank correlation test revealed some significant associations between myonectin serum level (Table 3).

\section{Discussion}

In this study, no significant change was observed in the myonectin serum level after short-term PhA. Myonectin before PhA was also associated with weight, BMI, serum creatinine and uremic acid. The groups of men and women differed significantly in age, height, weight, BMI, HDL-C, serum creatinine, uremic acid and myonectin before PhA levels. Myonectin is secreted by skeletal muscles. Skeletal muscle mass in young, healthy adults is positively correlated with weight, BMI. Moreover, serum creatinine and uremic acid levels are dependent on muscle mass [10-11]. These associations may provide a rationale for the observed results.

Myonectin in response to PhA increases the expression of CD36, fatty acid transport proteins (FATP), and fatty acid binding proteins (FABP) in hepatocytes and adipocyte, resulting in increasing FFA uptake [2]. It is known that myonectin is associated with IR [4-5] and circulating FFA induces IR [12]. It is possible that myonectin is secondarily upregulated in the IR state in order to diminish circulating FFA levels. In a young, 
Table 1. General characteristics of the study group

\begin{tabular}{|c|c|c|c|c|}
\hline Feature [units] & median (IQR) / n (\%) & $\begin{array}{c}\text { Female }(n=19) \\
\text { median (IQR) / } n(\%)\end{array}$ & $\begin{array}{c}\text { Male }(n=10) \\
\text { median (IQR) / } n(\%)\end{array}$ & p-value \\
\hline Smokers & $4(13.8)$ & $2(10) .5$ & $2(20.0)$ & 0.43 \\
\hline Age [years] & $22(20-23)$ & $21(20-22)$ & $23(22-23)$ & $<0.01$ \\
\hline Height [m] & $1.72(16.5-1.76)$ & $1.69(1.64-1.72)$ & $1.82(1.76-1.85)$ & $<0.001$ \\
\hline Weight [kg] & $59(54-78)$ & $54(53-59)$ & $80(78-86)$ & $<0.001$ \\
\hline BMI $\left[\mathrm{kg} / \mathrm{m}^{2}\right]$ & $21.3(19.4-24.0)$ & $20.1(18.5-21.3)$ & $24.0(23.8-25.7)$ & $<0.001$ \\
\hline Fasting Glucose [mmol/] & $5.1(4.8-5.3)$ & $5.1(4.8-5.3)$ & $5.1(4.6-5.4)$ & 0.80 \\
\hline Fasting insulin $[\mu \mathrm{lU} / \mathrm{ml}]$ & $16.1(12.5-19.1)$ & $16.7(12.1-18.6)$ & $15.2(12.8-19.6)$ & 0.79 \\
\hline HOMA-IR & $3.7(2.8-4.3)$ & $3.7(2.6-4.2)$ & $3.4(3.0-4.3)$ & 0.98 \\
\hline Total Cholesterol [mmol/l] & $4.4(3.9-5.2)$ & $4.4(4.2-5.4)$ & $4.0(3.7-4.6)$ & 0.07 \\
\hline HDL-C [mmol/l] & $1.6(1.4-1.9)$ & $1.7(1.5-2.1)$ & $1.4(1.2-1.7)$ & 0.04 \\
\hline LDL-C [mmol/l] & $2.3(2.0-3.0)$ & $2.3(2.0-3.1)$ & $2.2(1.8-2.5)$ & 0.35 \\
\hline Triglycerides [mmol/] & $0.84(0.61-1.1)$ & $0.85(0.63-1.1)$ & $0.70(0.48-0.98)$ & 0.33 \\
\hline Serum Creatinine $[\mu \mathrm{mol} / \mathrm{l}]$ & $70.4(70.3-79.6)$ & $70.7(61.9-70.7)$ & $88.4(79.6-97.3)$ & $<0.001$ \\
\hline Uremic Acid $[\mu \mathrm{mol} / \mathrm{l}]$ & $279.6(243.9-339.0)$ & $243.9(214.1-279.6)$ & $358.9(339.0-395.5)$ & $<0.001$ \\
\hline $\begin{array}{l}\text { METmax during Treadmill Test } \\
{[\mathrm{kcal} / \mathrm{kg} / \mathrm{h}]}\end{array}$ & $11.8(10.4-14.3)$ & $11.5(10.4-14.0)$ & $13.9(11.8-14.8)$ & 0.23 \\
\hline IPAQ Total Weekly MET $[\mathrm{kcal} / \mathrm{kg} / \mathrm{h}]$ & $3448(1794-4730)$ & $3842(1794-5205)$ & $2669(1328-3900)$ & 0.11 \\
\hline Myonectin before PhA [ng/ml] & $0.67(0.14-2.9)$ & $0.15(0.14-1.09)$ & $3.92(2.24-5.30)$ & 0.02 \\
\hline Myonectin after $\mathrm{PhA}[\mathrm{ng} / \mathrm{ml}]$ & $1.08(0.15-2.44)$ & $0.56(0.15-1.75)$ & $2.43(0.78-4.00)$ & 0.09 \\
\hline Change of the Myonectin Serum Level & $0.01([-0.84]-0.67)$ & $0.01([-0.17]-0.95)$ & $-0.65([-3.38]-0.67)$ & 0.10 \\
\hline
\end{tabular}

BMI — Body Mass Index, HDL-C — High-Density Lipoprotein Cholesterol, HOMA-IR — Homeostatic Model Assessment Measuring Insulin Resistance, IPAQ - The International Physical Activity Questionnaire, IQR — Interquartile Range, LDL — Low-Density Lipoprotein Cholesterol, MET — Metabolic Equivalent of Task, METmax — Maximal MET

Table 2. Wilcoxon test outcomes

\begin{tabular}{lccr}
\hline & $\begin{array}{c}\text { Before Physical Activity median } \\
\text { (IQR) }\end{array}$ & $\begin{array}{c}\text { After Physical Activity median } \\
\text { (IQR) }\end{array}$ & p value \\
\hline Myonectin [ng/ml] & $0.67(0.14-2.9)$ & $1.08(0.15-2.44)$ & 0.84 \\
Systolic Blood Pressure [mmHg] & $115(110-124)$ & $120(112-124)$ & 0.20 \\
Diastolic Blood Pressure [mmHg] & $75(70-80)$ & $72(70-80)$ & 0.28 \\
Heart Rate [bpm] & $82(78-93)$ & $96(91-100)$ & $<0.001$ \\
\hline
\end{tabular}

healthy population with no presence of metabolic syndrome, the release of myonectin might be too low and/or slow in comparison with patients with IR to observe any significant change in myonectin serum level in 6 minutes after short-term PhA.

Interestingly, the change in the myonectin serum level after short-term PhA was negatively associated with both insulin in fasting state and HOMA-IR. Toloza et al. studied 81 non-diabetic adults and assessed the association between insulin resistance direct and indirect markers and myokines [4]. Fasting myonectin adjusted to BMI, age and sex were found to be negativelyassociated with the Insulin Sensitivity Index (standardized beta $=-0.235, p=0.023)$. The authors did not report any significant association with HOMA-IR. Lim et al. investigated the effects of 1-h per week of aerobic physical activity lasting 10-weeks on myonectin and insulin resistance in 14 young (22.5 \pm 2.7 years) and 14 older $(60.3 \pm 5.2$ years) women [5]. Before the training apositive correlation between HOMA-IR and myonectin serum level was found only in older women in fasting state at rest $(r=0.35 ; p<0.05)$. After 10 -weeks of training, changes in HOMA-IR and changes in myonectin were positively correlated $(r=0.462 ; p<0.01)$ in both groups. Both the aforementioned studies demonstrated a positive dependence between insulin resistance and 
Table 3. Associations between study features and myonectin serum level

\begin{tabular}{|c|c|c|c|c|c|c|}
\hline \multirow[t]{2}{*}{ Feature [units] } & \multicolumn{2}{|c|}{$\begin{array}{c}\text { Myonectin Before Physical } \\
\text { Activity [ng/ml] }\end{array}$} & \multicolumn{2}{|c|}{$\begin{array}{c}\text { Myonectin After Physical } \\
\text { Activity [ng/ml] }\end{array}$} & \multicolumn{2}{|c|}{$\begin{array}{l}\text { Change of the Myonectin } \\
\text { Serum Level }[\mathrm{ng} / \mathrm{ml}]\end{array}$} \\
\hline & Rs & p-value & Rs & p-value & Rs & p-value \\
\hline Age [years] & 0.33 & 0.08 & 0.24 & 0.22 & -0.14 & 0.47 \\
\hline Height [m] & 0.30 & 0.11 & 0.35 & 0.07 & -0.08 & 0.68 \\
\hline Weight [kg] & 0.38 & 0.04 & 0.21 & 0.29 & -0.41 & 0.03 \\
\hline BMI [kg/m²] & 0.40 & 0.03 & 0.19 & 0.31 & -0.47 & 0.01 \\
\hline Fasting Glucose [mmol/] & 0.07 & 0.71 & 0.13 & 0.50 & -0.03 & 0.88 \\
\hline Fasting insulin $[\mu \mathrm{lU} / \mathrm{ml}]$ & 0.09 & 0.64 & -0.25 & 0.19 & -0.38 & 0.04 \\
\hline HOMA-IR & 0.15 & 0.45 & -0.21 & 0.29 & -0.43 & 0.02 \\
\hline Total Cholesterol [mmol/l] & -0.01 & 0.95 & 0.19 & 0.32 & 0.20 & 0.30 \\
\hline $\mathrm{HDL}-\mathrm{C}[\mathrm{mmol} / \mathrm{l}]$ & -0.21 & 0.28 & 0.04 & 0.84 & 0.21 & 0.28 \\
\hline LDL-C [mmol/l] & 0.13 & 0.49 & 0.30 & 0.11 & 0.15 & 0.44 \\
\hline Triglycerides [mmol/] & -0.12 & 0.55 & -0.35 & 0.06 & -0.23 & 0.22 \\
\hline Serum Creatinine $[\mu \mathrm{mol} / \mathrm{l}]$ & 0.40 & 0.03 & 0.32 & 0.09 & -0.32 & 0.09 \\
\hline Uremic Acid $[\mu \mathrm{mol} / /]$ & 0.41 & 0.03 & 0.43 & 0.02 & -0.17 & 0.38 \\
\hline $\begin{array}{l}\text { METmax during Treadmill } \\
\text { Test }[\mathrm{kcal} / \mathrm{kg} / \mathrm{h}]\end{array}$ & 0.16 & 0.41 & 0.04 & 0.84 & -0.20 & 0.30 \\
\hline $\begin{array}{l}\text { IPAQ Total Weekly MET } \\
{[\mathrm{kcal} / \mathrm{kg} / \mathrm{h}]}\end{array}$ & -0.06 & 0.78 & -0.07 & 0.71 & 0.01 & 0.10 \\
\hline
\end{tabular}

BMI — Body Mass Index, HDL-C — High-Density Lipoprotein Cholesterol, HOMA-IR — Homeostatic Model Assessment Measuring Insulin Resistance, IPAQ — The International Physical Activity Questionnaire, IQR — Interquartile Range, LDL — Low-Density Lipoprotein Cholesterol, MET — Metabolic Equivalent of Task, METmax — Maximal MET

myonectin. In our study, no such association was found. However, the negative association between changes in the myonectin serum level after PhA and simultaneously no significant change in myonectin serum level after $\mathrm{PhA}$ is intriguing. The change in myonectin serum level is highest among young volunteers with the lowest HOMA-IR value in the fasting state. It is possible that in those groups an increase of myonectin is more rapid than in the group characterized by higher HOMA-IR values. However, this possible phenomenon requires more detailed studies.

This study has several limitations. Firstly, we included only 29 volunteers in the study. Secondly, no additional data such as body composition or FFA were collected. Body composition analysis may reveal a potential association between myonectin serum level and muscle mass. Finally, we have not collected blood samples after a longer period of time than 6 minutes.

In conclusion, myonectin serum concentration does not change after short-term physical activity among young, healthy people. Myonectin serum level may be associated with muscle mass in a young, healthy population. Changes in myonectin serum level after short-term physical activity may be associated with fasting insulin resistance.

\section{Disclosure}

None of the authors declared any potential conflict of interests.

\section{Funding}

This work was supported by a Student's grant of the Student Scientific Society, Poznan University of Medical Sciences.[140/2016]

Contribution:

Concept - CM, KarM, Data collection - KamM, JK, GA, SJ, KarM, CM, Laboratory analysis - LB, WA, Statistical analysis - KamM, Draft preparation - KamM, Final approval —KamM, JK, GA, SJ, KarM, LB, WA, CM

\section{List of abbreviations:}

FFA - Free Fatty Acids

IPAQ - The International Physical Activity Questionnaire

IR — Insulin Resistance

MET - Metabolic Equivalent of the Task

PhA - Physical Activity 


\section{References}

1. Park SY, Choi JH, Ryu HSu, et al. C1q tumor necrosis factor alpha-related protein isoform 5 is increased in mitochondrial DNA-depleted myocytes and activates AMP-activated protein kinase. J Biol Chem. 2009; 284(41): 27780-27789, doi: 10.1074/jbc.M109.005611, indexed in Pubmed: 19651784.

2. Seldin MM, Peterson JM, Byerly MS, et al. Myonectin (CTRP15), a novel myokine that links skeletal muscle to systemic lipid homeostasis. J Biol Chem. 2012; 287(15): 11968-11980, doi: 10.1074/jbc.M111.336834, indexed in Pubmed: 22351773.

3. Sharma N, Castorena CM, Cartee GD. Greater insulin sensitivity in calorie restricted rats occurs with unaltered circulating levels of several important myokines and cytokines. Nutr Metab (Lond). 2012; 9(1): 90, doi: 10.1186/1743-7075-9-90, indexed in Pubmed: 23067400

4. Toloza FJK, Mantilla-Rivas JO, Pérez-Matos MC, et al. Plasma Levels of Myonectin But Not Myostatin or Fibroblast-Derived Growth Factor 21 Are Associated with Insulin Resistance in Adult Humans without Diabetes Mellitus. Front Endocrinol (Lausanne). 2018; 9: 5, doi: 10.3389/fendo.2018.00005, indexed in Pubmed: 29445355

5. Lim S, Choi SH, Koo BoK, et al. Effects of aerobic exercise training on C1q tumor necrosis factor $\alpha$-related protein isoform 5 (myonectin): association with insulin resistance and mitochondrial DNA density in women. J Clin Endocrinol Metab. 2012; 97(1): E88-E93, doi: 10.1210/jc.2011-1743, indexed in Pubmed: 22031510

6. Koivisto V, Yki-Järvinen H, DeFronzo R. Physical training and insulin sensitivity. Diabetes / Metabolism Reviews. 1986; 1(4): 445-481, doi: 10.1002/dmr.5610010407.

7. Hagströmer M, Oja P, Sjöström M. The International Physical Activity Questionnaire (IPAQ): a study of concurrent and construct validity. Public Health Nutr. 2006; 9(6): 755-762, indexed in Pubmed: 16925881.

8. Matthews DR, Hosker JP, Rudenski AS, et al. Homeostasis model assessment: insulin resistance and beta-cell function from fasting plasma glucose and insulin concentrations in man. Diabetologia. 1985; 28(7): 412-419, indexed in Pubmed: 3899825.

9. Bruce R. Methods of exercise testing. The American Journal of Cardiology. 1974; 33(6): 715-720, doi: 10.1016/0002-9149(74)90211-2.

10. Baxmann AC, Ahmed MS, Marques NC, et al. Influence of muscle mass and physical activity on serum and urinary creatinine and serum cystatin C. Clin J Am Soc Nephrol. 2008; 3(2): 348-354, doi: 10.2215/CJN.02870707, indexed in Pubmed: 18235143.

11. Maiuolo J, Oppedisano F, Gratteri S, et al. Regulation of uric acid metabolism and excretion. Int J Cardiol. 2016; 213: 8-14, doi: 10.1016/j. ijcard.2015.08.109, indexed in Pubmed: 26316329.

12. Capurso C, Capurso A. From excess adiposity to insulin resistance: the role of free fatty acids. Vascul Pharmacol. 2012; 57(2-4): 91-97, doi: 10.1016/j.vph.2012.05.003, indexed in Pubmed: 22609131 